\title{
Refractive Index Anisotropy and Diffusion Rate in Cartilage Tissue
}

\section{Georgy V. Simonenko}

N. G. Chernyshevsky Saratov National Research State University, 83 Astrakhanskaya str., Saratov 410012, Russia

e-mail: gvsim1960@hotmail.com

\begin{abstract}
The paper presents the results of experimental and theoretical studies of cartilage tissue optical characteristics after the immersion impact and without it. We have found that the immersion effect leads to structural changes in the cartilage tissue, in particular, to the increased size of the scattering elements. The refractive index anisotropy of the cartilage tissue is measured in the samples subjected to immersion impact and without it. We demonstrate theoretically that the diffusion kinetics of the immersion fluid in the biotissue strongly depends upon the anisotropy of the diffusion rate along the collagen fibres and in the perpendicular direction. Due to the diffusion anisotropy, the increase of the mean rate of the immersion fluid diffusion in the biotissue can lead to the fall of light transmission through the sample rather than to its expected increase. (C) 2017 Journal of Biomedical Photonics \& Engineering.
\end{abstract}

Keywords: optical properties; cartilage tissue; diffusion; immersion clearing.

Paper \#3224 received 21 Jun 2017; revised manuscript received 4 Sep 2017; accepted for publication 4 Sep 2017; published online 25 Sep 2017. doi: 10.18287/JBPE17.03.030302.

\section{References}

1. V. V. Tuchin, "Tissue optics and photonics: light-tissue interaction," Journal of Biomedical Photonics \& Engineering 1(2), 98-134 (2015).

2. A. Bykov, T. Hautala, M. Kinnunen, A. Popov, S. Karhula, S. Saarakkala, M. T. Nieminen, V. Tuchin, and I. Meglinski, "Imaging of subchondral bone by optical coherence tomography upon optical clearing of articular cartilage," Journal of Biophotonics 9(3), 270-275 (2016).

3. V. V. Tuchin (Ed.), Coherent-Domain Optical Methods: Biomedical Diagnostics, Environmental Monitoring and Material Science, vol. 2, 2nd edition, Springer-Verlag, NY (2013).

4. S. M. Daly, and M. J. Leahy, "Go with the flow: a review of methods and advancements in blood flow imaging," Journal of Biophotonics 6(3), 217-255 (2013).

5. M. G. Ghosn, V. V. Tuchin, and K. V. Larin, "Non-destructive quantification of analytes diffusion in cornea and sclera by using optical coherence tomography," Investigative Opthalmology \& Visual Science 48(6), 2726-2733 (2007).

6. E. A. Genina, A. N. Bashkatov, E. A. Kolesnikova, M. V. Basko, G. S. Terentyuk, and V. V. Tuchin, "Optical coherence tomography monitoring of enhanced skin optical clearing in rats in vivo," Journal of Biomedical Optics 19(2), 021109 (2013).

7. V. V. Tuchin, "Polarized light interaction with tissues," Journal of Biomedical Optics 21(7), 071114 (2016).

8. D. Zhu, K. V. Larin, Q. Luo, and V. V. Tuchin, "Recent progress in tissue optical clearing", Laser \& Photonics Reviews 7(5), 732-757 (2013).

9. J. Wang, Y. Zhang, T. H. Xu, Q. M. Luo, and D. Zhu, "An innovative transparent cranial window based on skull optical clearing," Laser Physics Letters 9(6), 469-473 (2012).

10. E. A. Genina, A. N. Bashkatov, Yu. P. Sinichkin, I. Yu. Yanina, and V. V. Tuchin, "Optical clearing of biological tissues: prospects of application in medical diagnostics and phototherapy," Journal of Biomedical Photonics \& Engineering 1(1), 22-58 (2015).

11. E. A. Genina, A. N. Bashkatov, M. D. Kozintseva, and V. V. Tuchin, "OCT study of optical clearing of muscle tissue in vitro with 40\% glucose solution," Optics and Spectroscopy 120(1), 20-27 (2016). 
12. C.-H. Liu, M. Singh, J. Li, Z. Han, C. Wu, S. Wang, R. Idugboe, R. Raghunathan, E. N. Sobol, V. V. Tuchin, M. Twa, and K. V. Larin, "Quantitative assessment of hyaline cartilage elasticity during optical clearing using optical coherence elastography," Modern Technologies in Medicine 7(1), 44-51 (2015).

13. E. A. Genina, A. N. Bashkatov, and V. V. Tuchin, "Tissue optical immersion clearing," Expert Review of Medical Devices 7(6), 825-842 (2010).

14. K. V. Larin, M. G. Goshn, A. N. Bashkatov, E. A. Genina, N. A. Trunina, and V. V. Tuchin, "Optical clearing for OCT image enhancement and in-depth monitoring of molecular diffusion," IEEE Journal of Selected Topics in Quantum Electronics 18(3), 1244-1259 (2012).

15. M. G. Ghosn, V. V. Tuchin, and K. V. Larin, "Depth-resolved monitoring of glucose diffusion in tissues by using optical coherence tomography," Optics Letters 31(15), 2314-2316 (2006).

16. O. I. Baum, A. I. Omel'chenko, I. O. Ryzhkov, M. V. Obrezkova, V. V. Lunin, and E. N. Sobol', "Effect of Omnipaque on the optical properties and laser-induced changes in the thermostability of nucleus pulposus of the intervertebral disk," Doklady Biochemistry and Biophysics 428, 261-263 (2009).

17. V. L. Vesnin, and V. G. Muradov "Spectrophotometric complex based on the monochromator MDR-41 for studying the absorption spectra in the range 400-1800 nm," Izvestiya Samarskogo nauchnogo tsentra Rossiiskoy Akademii Nauk 10(3), 724-731 (2008) [in Russian].

18. D. B. Judd, and G. Wyszecki, Color in Business, Science and Industry, $2^{\text {nd }}$ ed., John Wily \& Sons, NY (1975).

19. G. V. Simonenko, V. V. Tuchin, and N. A. Lakodina, "Measurement of the optical anisotropy of biological tissues with the use of a nematic liquid crystal cell," Journal of Optical Technology 67(6), 559-561 (2000).

20. V. Chigrinov, H. S. Kwok, D. Yakovlev, G. Simonenko, and V. Tsoi, "LCD optimization and modeling," Journal of the Society for Information Display 12(2), 183-187 (2004).

21. E. S. Bukareva, G. V. Simonenko, and V. V. Tuchin, "Features of the kinetics of the immersion clarification of biological tissue," Journal of Optical Technology 80(2), 119-123 (2013).

22. G. A. Korn, and T. M. Korn, Mathematical Handbook for Scientists and Engineers, McGraw-Hill. Book Co., New York (1968).

23. Yu. Ya. Kuzyakiv, K. A. Semenenko, and N. B. Zorov, Methods of Spectral Analysis, Izd-vo MGU, Moscow (1990) [in Russian].

24. D. A. Zimnyakov, G. V. Simonenko, and V. V. Tuchin, "Dispersion dependence of the optical anisotropy and the degree of depolarization of fibrous tissues," Journal of Optical Technology 77(9), 577-581 (2010).

25. K. V. Berezin, K. N. Dvoretskiy, M. L. Chernavina, V. V. Nechaev, A. M. Likhter, I. T. Shagautdinova, E. Yu. Stepanovich, O. N. Grechukhina, and V. V. Tuchin, "Studying the mechanism of tissue optical clearing using the method of molecular dynamics," Proc. SPIE 10336, 103360J (2017).

26. A. N. Bashkatov, E. A. Genina, T. G. Kamenskikh, et al., "Study of mildronate diffusion in th human eye sclera," Izvestiya Saratovskogo universiteta. Novaya seriya. Seriya Fizika 16(3), 167-177 (2016) [in Russian].

27. A. A. Ovchinnikov, S. F. Timashev, and A. A. Belyi, Kinetics of Diffusion-Controlled Chemical Processes, Khimiya, Moscow (1986) [in Russian].

\section{Introduction}

The diseases of musculoskeletal system, in which cartilage tissues are of particular importance, are widespread at present time. The arthrous cartilage is a biotissue playing a very important role in the normal functioning of joints, reducing the loads and providing the mobility. The condition of the cartilage tissue has to be determined for the diagnostics and treatment of various pathologies. At present, more and more optical diagnostic methods are used for this purpose. Optical diagnostics is promising because of its noninvasiveness, high functionality and high spatial resolution [1]. During the last decade the methods of optical coherence tomography (OCT) have been intensely developed and widely used in a number of applications [2, 3], including the visualisation of spatial distribution of blood vessels in human skin [4] and visualisation of molecular diffusion in biological tissues $[5,6]$. For the diagnostics of cartilage tissue, the polarisation-sensitive OCT was used [7]. Essential enhancement of the OCT image contrast and increased probing depth can be achieved by applying the immersion clearing technique [8-11] to the studied samples, including those of the cartilage tissue [1, 12]. The principles of immersion clearing are based on the partial replacement of the interstitial fluid by an immersion agent and the partial tissue dehydration, which matches the refractive indices of collagen and interstitial fluid, as well as enhances the ordering of the tissue structural elements and, consequently, reduces the scattering of light by a biotissue [13-15]. As a rule, the random arrangement of the scattering structures in a biotissue causes fast depolarisation of the probing radiation. For this reason, many methods of optical diagnostics do not take the polarisation effects into account [5, 6, 8-11]. However, the polarisation effects can play a certain role in the probing of relatively thick samples of biotissue with polarised radiation, which can be characterised quantitatively by recording the degree of depolarisation of the transmitted light or the transformation of its initial polarisation state [7, 15]. Thus, the problem is how the anisotropy of a tissue (in 
particular, cartilage) affects the diffusion kinetics of the tissue immersion clearing and how the immersion agent affects the biotissue structure and, therefore, the refractive index anisotropy. The latter can play an essential role in the development of polarisationsensitive OCT [7]. The goal of the present paper is to determine the role of the tissue anisotropy in the kinetics of its immersion clearing and to estimate the structural changes of the tissue in this process. The study included the solution of the following problems: to estimate the effect of the immersion agent on the structure of the cartilage tissue, to estimate the cartilage refractive index anisotropy in the samples after immersion and the control ones, and to reveal the role of the diffusion rate anisotropy in the kinetics of the biotissue immersion clearing.

In the present paper, we report the results of studying the spectral characteristics (in the range from 400 to $1400 \mathrm{~nm}$ ) and optical anisotropy properties of the cartilage tissue after the immersion clearing, as well as the kinetics of immersion agent diffusion in the cartilage with its anisotropy properties taken into account. The first part of the paper is devoted to the study of transmission spectra of the cartilage tissue affected by the immersion fluid and the determination of the cartilage refractive index anisotropy. The second part of the paper presents the results of computer simulation of the immersion fluid diffusion in the cartilage tissue with the structural anisotropy of the tissue taken into account.

\section{Methods and materials}

For the study, we used the samples of porcine ear cartilage. Before starting the experiment, the biotissue was cut into samples with the area of about $20 \times 15$ $\mathrm{mm}^{2}$, in which the bundles of collagen fibres were parallel to the surface. Some samples were prepared from the right ear and some from the left ear of the same pig. The samples were divided into two groups, one of them subjected to the immersion with Omnipaque ${ }^{\mathrm{TM}}$ agent [16] by placing the biotissue sample into the liquid for a day. The second group was left intact for control. In each group, there were five samples. Then all samples were dried in air at the room temperature during three days to remove the free interstitial fluid from them. Before and after drying the thickness of the samples was measured with the accuracy of $\pm 10 \mu \mathrm{m}$ at a few points and then averaged. Before drying the mean thickness of the studied samples amounted to $2.3 \pm 0.18$ $\mathrm{mm}$ in the first group and $0.8 \pm 0.05 \mathrm{~mm}$ in the second one. After drying the mean thickness of the studied samples was $1.5 \pm 0.18 \mathrm{~mm}$ in the first group and $0.5 \pm 0.05 \mathrm{~mm}$ in the second one. To improve the accuracy of the measurements, the thinner samples of the cartilage were used without immersion, and the thicker ones to study the consequences of immersion clearing. No additional processing of the samples was undertaken. The spectra of collimated transmission and the refractive index anisotropy of the biotissue samples were experimentally studied.
The transmission measurements were performed using the spectral system based on the MDR-41 monochromator, analogous to the system described in detail in Ref. [17]. As a radiation source, the halogen capsule lamp with the spectral range from 340 to 2500 $\mathrm{nm}$ was used. The transmission was measured with the spectral step of $1 \mathrm{~nm}$. The limit resolution interval of the monochromator did not exceed $0.02 \mathrm{~nm}$. The sample was illuminated with a parallel light beam $1 \mathrm{~mm}$ in diameter. Immediately after the sample the aperture having the diameter $1 \mathrm{~mm}$ was installed. The output slit of the monochromator was $10 \mu \mathrm{m}$ wide and $2 \mathrm{~mm}$ high. The relative error of the transmission measurement did not exceed $1 \%$. To obtain the transmission spectra the standard commercial MdrWin software operating under OS Windows and supplied together with the monochromator was used.

To measure the refractive index anisotropy, we used the method of visual colour determination (method A of Ref. [18]), based on the comparison of the etalon colour with the current colour of the sample. The relative error of this method does not exceed $2 \%$. To analyse the polarised beam interference image colour in the biotissue samples, we used the polarised light microscope POLAM-P11 with $4 \times$ objective and digital camera DCM-500 connected to the computer. To determine the sample colour by the colour of the etalon we used the method of comparison, described in Ref. [19] and the software package MOUSE-LCD having the computation accuracy not worse than 5\% [20]. The choice of the liquid crystal (LC) cell as an etalon is caused by the possibility of easy optical anisotropy variation of the liquid crystal by applying small electric voltage (from 0 to $10 \mathrm{~V}$ ) to the control electrodes of the cell. In our case, a slightly modified technique of measuring the biotissue optical anisotropy was used, its main difference consisting in exploitation of a virtual LC cell. On the PC monitor, one window of any graphic editor displayed the image of the polarised beams interference, obtained by means of the polarised light microscope and the digital camera. The comparison window (etalon window) displayed the polarisation beams interference image in the virtual planar nematic liquid crystal cell. The virtual liquid crystal cell was simulated using the computer software MOUSE-LCD [20]. Given the thickness of the virtual LC cell $d$, the main physical parameters of the liquid crystal $(\Delta n$ being the LC optical anisotropy), and the value of the control voltage on the cell electrodes, we calculated the value $\Delta n d$ for the liquid crystal and the colour characteristics of the virtual cell. By changing the thickness of the liquid crystal layer, we achieved similar visual perception of the virtual liquid crystal cell image and the image of the biotissue sample. The similar visual perception of the etalon and sample was assumed to mean that the colour coordinated (i.e., the colour) of the sample and the etalon are similar, too. Since the change of the colour coordinates of the biotissue and the liquid crystal is caused only by the interference of polarised beams, for similar values of $\Delta n d$ the transmission 
spectra should be similar. Thus, we determined $\Delta n d$ for the biotissue sample.

To study the kinetics anisotropy of the immersion fluid diffusion in the biotissue we used the method of computer simulation described in detail in Ref. [21]. Briefly, the essence of the method is the following. The problem of the immersion fluid diffusion in the biotissue sample is solved basing on the simple diffusion model, in which the difference of diffusion rates along the collagen fibres and in the perpendicular direction is taken into account. Let $\mathrm{C}$ be the concentration of the immersion fluid depending on the time $t$ and three spatial coordinates $x$ (inward), $y$ (across the fibres and parallel to the surface) and $z$ (along the fibres). The diffusion equation with three spatial coordinates has the form [21]:

$$
\begin{aligned}
& \frac{\partial C(x, y, z, t)}{\partial t}=v_{x}(x, y, z) \frac{\partial^{2} C(x, y, z, t)}{\partial x^{2}}+ \\
& +v_{y}(x, y, z) \frac{\partial^{2} C(x, y, z, t)}{\partial y^{2}}+v_{z}(x, y, z) \frac{\partial^{2} C(x, y, z, t)}{\partial z^{2}}
\end{aligned}
$$

where $\mathrm{v}_{x}, \mathrm{v}_{y}, \mathrm{v}_{z}$ are the immersion liquid diffusion rates along the respective coordinate axes. form

Let the initial condition at $t=0$ be specified in the

$$
C(x, y, z, 0)=\varphi(x, y, z)
$$

where $\varphi(x, y, z)$ is the surface distribution of the immersion fluid at the initial moment of time at the point of the clearing agent application.

In this model, the nonstationary, inhomogeneous, and anisotropic diffusion of the immersion fluid in the biotissue sample is considered. Assume that the immersion fluid can enter the sample from one side either all over the surface, or only in the centre of the sample, and the application domain has the shape of either a rectangle (the entire sample surface), or a circle with the uniform concentration. The biotissue is represented by a system of long cylindrical inclusions (collagen fibres), surrounded by the base substance. The characteristic geometric parameters of the inclusions are the length $l_{0}$, the diameter $d_{0}$, the separation $a_{0}$ between the inclusions along the axes $x$ or $y$, the separation $b_{0}$ between the inclusions along the axis $z$, and the deviation angle $\varphi$ between the inclusion and the $z$-axis, specified arbitrarily for each individual inclusion. The maximal possible deviation from the axis is an input parameter. To calculate the diffusion rate, we introduce the probability $p$ that the diffusion at the considered point will go in a certain direction. Inside the collagen fibres, the probability is taken to be close to zero, since the collagen is much denser than the surrounding medium, and the molecular diffusion inside the fibres is hampered. If the considered point is in the base substance, and there is the base substance in the chosen direction, too, the diffusion probability is taken to be close to one. For each direction the coefficients of diffusion are recalculated at each point, namely, depending on the coordinate, the diffusion coefficient is multiplied by $p$. We solve Eq. (1) using the finitedifference method and the explicit scheme [22], assuming the diffusion rates along the $x$-axis and the $y$ axis constant or expressed in the known analytic form. For this purpose, we approximate the second derivatives in Eq. (1) by the ratios of finite differences, which yields the finite-difference equation:

$$
\begin{aligned}
& \frac{c_{i j m}^{k+1}-c_{i j m}^{k}}{h_{4}}=v_{x} \frac{c_{i+1, j m}^{k}-2 c_{i j m}^{k}+c_{i-1, j m}^{k}}{h_{1}^{2}}+ \\
& +v_{y} \frac{c_{i, j+1, m}^{k}-2 c_{i j m}^{k}+c_{i, j-1, m}^{k}}{h_{2}^{2}}+ \\
& +v_{z} \frac{c_{i j, m+1}^{k}-2 c_{i j m}^{k}+c_{i j, m-1}^{k}}{h_{3}^{2}}
\end{aligned}
$$

where $x_{i}=i h_{1}(i=0,1, \ldots), y_{i}=j h_{2}(j=0,1, \ldots)$, $z_{m}=j h_{3}(m=0,1, \ldots), t_{k}=k h_{4}(k=0,1, \ldots), h_{1}$ is the step in the direction $O x, h_{2}$ is the step in the direction $O y, h_{3}$ is the step in the direction $O z$, and $h_{4}$ is the step in time $t$. The values of the grid function at the nodes $\left(x_{i}, y_{j}, z_{m}, t_{k}\right)$ are denoted by the symbol $c_{i j m}^{k}$.

Eq. (3) can be written as a system of linear algebraic equations for the unknown values of the grid function in each layer.

In the zero layer the solution is found from the initial condition (2), presented as

$c_{i j m}^{0}=\varphi\left(x_{i}, y_{i}, z_{m}\right)$.

Thus, solving the system of Eq. (3) with the initial conditions (4) we get the spatiotemporal dependence of the immersion fluid concentration in the sample. The described technique is implemented in the programme for calculating the kinetics of the fluid diffusion in the biotissue with the tissue anisotropy taken into account.

Below we present the results of experimental and computer studies of different properties of the cartilage tissue.

\section{Optical properties of cartilage tissue}

One of the simple and efficient ways to increase the probing depth and improve the quality of imaging the tissue internal structures is to reduce the light scattering by means of optical clearing agents [1-3]. The immersion optical clearing is based on the impregnation of the tissue with the biocompatible chemical agent possessing sufficiently high refractive index and hyperosmotic properties. Penetrating into the sample and interacting with the biotissue components, the agent reduces the refractive index difference between the scatterers and the surrounding base medium and changes the relative position and size of the tissue structural elements due to dehydration [10-13]. Thus, it 
is possible to select three most probable mechanisms of the biotissue clearing, namely, the equalisation of refractive indices of the interstitial fluid and the collagen fibres, the change of collagen fibres packing density, and the size variation of the scatterers present in the biotissue. While the first mechanism is always present during the biotissue optical clearing, the second and the third ones require additional consideration. To eliminate the manifestations of the first mechanism, we dried all samples of the cartilage tissue before the measurements. Then we compared the optical characteristics (transmission spectra and refractive index anisotropy) of the samples subjected to the immersion before drying and the control samples, dried without immersion.

Fig. 1 presents the spectra of collimated transmission of the cartilage tissue samples after the immersion clearing and without it. For comparison, the transmission spectrum of the Omnipaque immersion agent itself is also shown. In the visible region, there is obviously no difference between the transmission spectra of the samples subjected to the immersion clearing and the control samples. In the infrared range, the observed situation is different. To analyse the spectra we used the differential spectroscopy method [23]. First, we see a different number of absorption peaks, the wavelengths of which are summarised in Table 1. The post-immersion cartilage tissue possesses six absorption peaks, while the intact tissue has only two peaks. The first absorption peak at $1000-1050 \mathrm{~nm}$ is nearly similar in all samples. The second peak in the samples subjected to the immersion manifests the own absorption Omnipaque agent. The common absorption peak at $1450-1500 \mathrm{~nm}$ is most probably due to the residual water. The rest three absorption peaks in the cartilage samples after immersion can be due to the structural changes of the biotissue induced by the immersing agent. Thus, the analysis of the transmission spectra shows that the application of immersion fluid changes the cartilage absorption spectrum in the infrared region and has no effect on the spectra in the visible range of optical radiation wavelengths. Therefore, the immersion fluid causes certain structural changes in the cartilage tissue.

Let us make some estimates of the cartilage tissue changes caused by the application of the immersion fluid. For these estimates, we chose the visible range, because in this range it is possible to perform independent polarisation measurements. For the measurements, we choose the transmission maximum for the samples with the immersion effect and without it. At the transmission maximum wavelength $(873 \mathrm{~nm})$ the ratio of scattering cross-section of the sample subjected to immersion $\sigma_{\mathrm{im}}$ and the control one $\sigma_{\mathrm{wi}}$ amounts to $\sigma_{\mathrm{im}} / \sigma_{\mathrm{wi}}=1.083$. Since in the cartilage tissue the light is scattered by collagen fibres, the scattering cross-section is a function of the packing density $f$ and diameter $d_{0}$ of the fibres. Thus, basing only on the transmission spectra of the cartilage tissue it is impossible to determine the changes produced in the biotissue by the immersion fluid. Therefore, we undertake independent measurements to answer this question.

To determine the refractive index anisotropy of the biotissue samples we exploited the polarised light microscope MIKMED, version 11. Using the method of colour comparison described above, we found that for the non-immersed sample $(\Delta n \cdot d) \approx 0.2 \mu \mathrm{m}$ and for the immersed one $(\Delta n \cdot d) \approx 0.6 \mu \mathrm{m}$. Here $\Delta n$ is the refractive index anisotropy in the biotissue and $d$ is the sample thickness. Keeping in mind that the mean thickness was $1.5 \mathrm{~mm}$ for the immersed samples and $0.5 \mathrm{~mm}$ for nonimmersed ones, we conclude that the in both cases the refractive index anisotropy is similar and amounts to nearly 0.0004 . This value of the cartilage tissue anisotropy agrees with the data reported in [24]. Since in the cartilage tissue the refractive index anisotropy is caused by the shape anisotropy [1, 7, 24], which depends upon the refractive indices of the interstitial fluid and the collagen fibres, as well as upon the packing density of the collagen fibres $f$, we can conclude that the immersion fluid does not affect the collagen packing density. From the comparison of transmission spectra of immersed and non-immersed samples and the data on the refractive index anisotropy in such samples, we can draw a conclusion that the action of immersion fluid changes the size of the collagen fibres.

Now we can estimate the change of the collagen fibre size under the action of the immersion fluid on the cartilage tissue. Usually the model of a scattering medium, including the cartilage tissue, is considered to consist of cylinders with the length much greater than the diameter [2]. Since the packing density of the collagen fibres $f$ is constant $\sigma_{\text {im }} / \sigma_{\mathrm{wi}}=\left(\mathrm{d}_{\mathrm{im}} / \mathrm{d}_{\mathrm{wi}}\right)^{2}, \mathrm{~d}_{\mathrm{im}}$ and $\mathrm{d}_{\mathrm{wi}}$ being the collagen fibre diameter in the immersed sample and in the non-immersed one, respectively. It follows that $\mathrm{d}_{\mathrm{im}} / \mathrm{d}_{\mathrm{wi}}=1.041$, i.e., the use of the immersion fluid increases the collagen fibre diameter by nearly $4 \%$. The reliability of the obtained data is worth a brief separate discussion. The accuracy of the measurements is comparable with that for the refractive index anisotropy (the relative error of the visual colour determination method is $2 \%$ ), and the accuracy of measuring the samples transmission spectra is essentially higher (the relative error does not exceed $1 \%$ ). Since for the numerical estimation of the structural changes we used the data of spectral measurements, and the colour ones were used only to ascertain the fact that the refractive index anisotropy is constant (and, therefore, the packing density of the fibres is unchanged), the fact that the diameter of collagen fibres increases can be considered reliably established. Moreover, the increase of the collagen fibres diameter in the process of biotissue immersion clearing was reported by the authors of Ref. [25], in which using the methods of molecular dynamics it was shown that the 


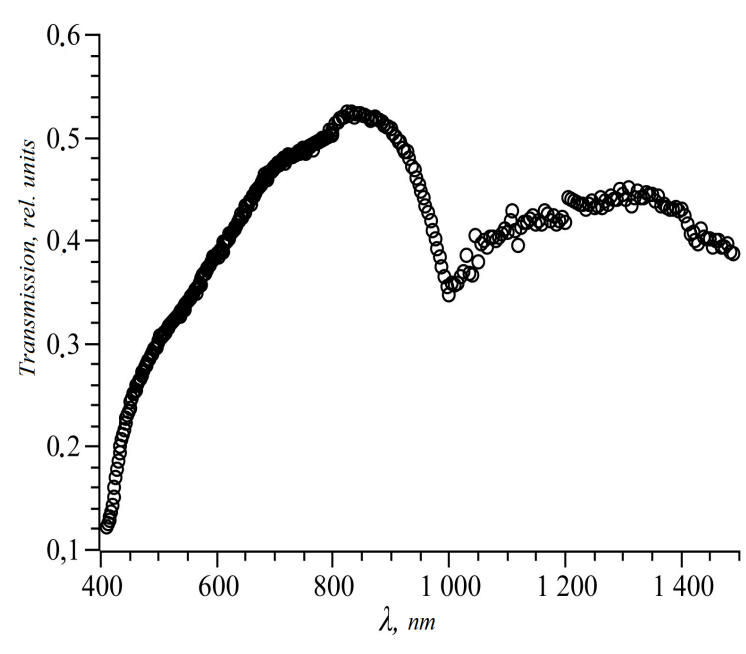

a

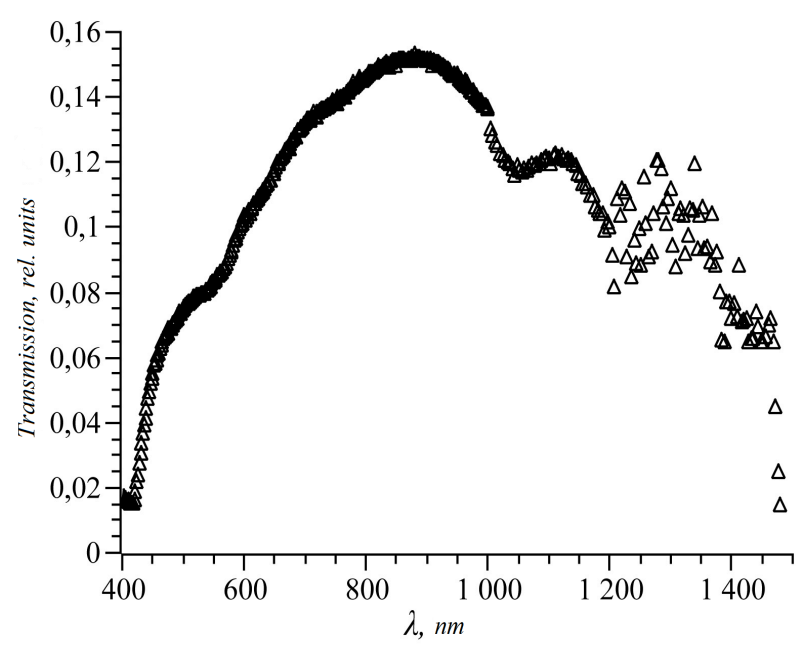

$\mathrm{b}$

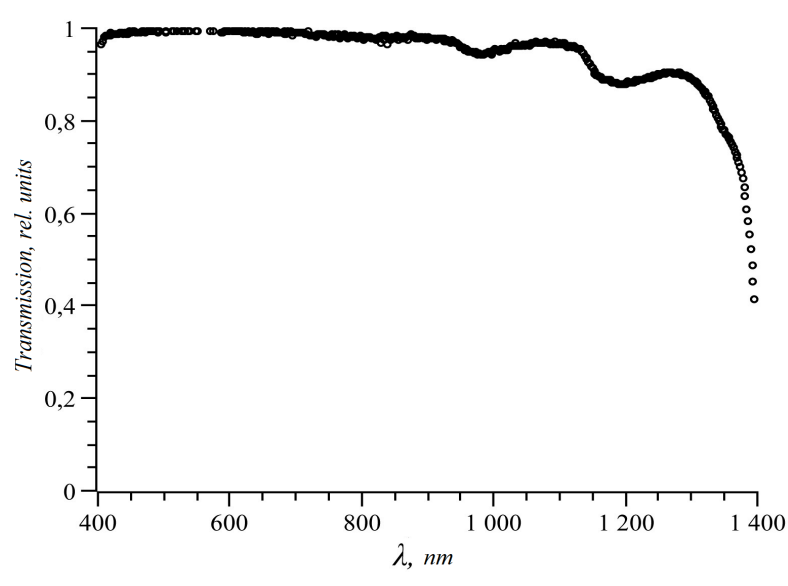

C

Fig. 1 Spectra of collimated transmission of the cartilage tissue without immersion treatment (a) and subjected to immersion (b); the spectrum of the immersion fluid itself (c).

Table 1 Positions of the absorption peaks for the cartilage tissue in the IR range.

Wavelength of light $\lambda, \mathrm{nm}$

\begin{tabular}{lccccc}
\hline $\begin{array}{l}\text { Cartilage tissue } \\
\text { after immersion }\end{array}$ & 1049 & 1207 & 1239 & 1307 & 1361 \\
\hline $\begin{array}{l}\text { Cartilage tissue } \\
\text { without immersion }\end{array}$ & 1003 & & & 1488 \\
\hline $\begin{array}{l}\text { Immersion fluid } \\
\text { Omnipaque }\end{array}$ & 1050 & 1200 & & 1507 \\
\hline
\end{tabular}

effect of the immersion fluid on a biotissue leads to the growth of the collagen fibres diameter.

Now let us briefly discuss the analysis of the cartilage tissue images produced by interference of polarised light beams in the biotissue samples. In this case, the sample transmission spectrum is known to depend upon the optical path length difference between the interfering beams and the angle between the optical axis of the sample and those of the polarisers. In our case, the studied samples were strongly nonuniform both in the layer thickness and in the orientation of collagen fibres that determine the orientation of the optical axis of the sample. However, although the variation of the layer thickness is large, as shown by our measurements, its impact on the transmission spectrum (colour) of the sample is small due to the minor value of the cartilage optical anisotropy $\Delta n \approx 0.0004$. Therefore, the different colour of the regions of cartilage tissue is 
caused only by the different orientation of collagen fibres (and the resulting difference of the optical axis orientation). This can be easily checked by rotating the microscope object stage through different angles that changes the colour of different regions to the complementary one. In our case, the light regions become dark and vice versa. Thick and thin samples show the same degree of homogeneity in the collagen fibres orientation. This observation means that the samples possess nearly the same number of overlapped layers with different orientation of the collagen fibres. As already mentioned above, the immersion agent does not affect the value of the cartilage tissue optical anisotropy.

\section{Diffusion of immersion fluid in the cartilage tissue}

As mentioned above, the improvement of the image contrast and the increase of the probing depth can be achieved by applying different immersion fluids that penetrate into the sample $[1,2]$. In this connection, it is interesting to study the kinetics of the immersion clearing process in different types of biotissues. Earlier we have studied the influence of the sample geometric parameters on the kinetics of the biotissue immersion clearing process [21]. The diffusion of different substances in a biotissue is often studied using purely optical techniques (see, e.g., [1, 3, 7, 26]), in which the influence of the clearing agent diffusion rate anisotropy (the difference of diffusion rates along the collagen fibres and in the perpendicular direction) and the sample geometry on the diffusion process itself remains unexplored. It is known [1] that, first of all, the geometric parameters of the light-scattering sample strongly affect the light attenuation coefficient, and, therefore, it is necessary to find out, which parameters affect only the diffusion of the immersion fluid and do not affect the optical properties, and which parameters affect both the diffusion kinetics and the attenuation coefficient. In this section of the paper, we report the computer analysis of the kinetics of the immersion fluid diffusion in a biotissue depending on the diffusion rate anisotropy with the fibrillar structure of the studied sample taken into account.

Consider the three-dimensional model of the biotissue immersion clearing, presented in the previous section. To describe the diffusion under the local application of the clearing agent the one-dimensional model has the following drawbacks. 1) In the frameworks of one-dimensional model of the clearing agent diffusion in the biotissue it is impossible to explain the nonmonotonic behavior of transmission as a function of clearing time without attracting other (different from diffusion) possible physical mechanisms that might take place [21]. 2) The determination of the diffusion coefficient of the clearing fluid in the onedimensional model can yield wrong diffusion rate, since in reality some time is always taken by the diffusion in three directions with different rates, whereas in the one- dimensional model the diffusion is considered to occur only into the depth of the sample. In our case, the model is characterised by the collagen fibre diameter, its length, the mean separation between the fibres and the mean angle of deviation of the fibres from a certain direction, as well as the diffusion rates along the fibres $v_{x}$ and in the perpendicular directions $v_{y}, v_{z}$. To simplify the analysis, we assumed that the sample is homogeneous in the $y$ and $z$ directions and $v_{y}=v_{z}$.

Fig. 2 illustrates the kinetics of the immersion fluid diffusion in the biotissue under the local application depending on the diffusion rates $v_{x}$ and $v_{y}$. The ordinate axis displays the value of the maximal possible concentration of the clearing fluid $C_{\max }$ at the fixed depth in the sample. Worth attention is the fact that the diffusion rate increase in the mutually perpendicular planes yields the opposite results. The explanation is that the increase of the diffusion rate $v_{x}$ along the fibres leads to the fluid spreading in this direction without penetration deep into the sample. Note that the dependence of $C_{\max }$ upon $\mathrm{v}_{x}$ or $\mathrm{v}_{y}$ is well described by linear functions, the parameters of which are presented in Fig. 2. The linear character of the dependences $C_{\max }\left(\mathrm{v}_{x}\right)$ and $C_{\max }\left(\mathrm{v}_{y}\right)$ follows from by the fact that the diffusion equation used in our studies is linear and the diffusion coefficients entering this equation are constant. The maximal possible value $C_{\max }$ depends on $v_{y}$ stronger than on $v_{x}$. The explanation is that with the increase of the diffusion rate across the fibres the immersion fluid more intensely penetrates deep into the sample, while the increase of the diffusion rate along the fibres the immersion fluid spreads over the sample and does not penetrate into it. That is why the increase of the mean rate $v\left(v=\left(v_{x}+v_{y}\right) / 2\right)$ of the diffusion of the immersion fluid in the biotissue can lead to the fall of light transmission instead of its increase, if the increase of the mean rate occurs at the expense of stronger diffusion along the fibres.

As shown by the simulation, the geometric factors (the size of collagen fibres, the separation between them, and the angles of the fibre orientations) weakly affect the kinetics of the immersion fluid diffusion under the local application. The explanation is that the size of the molecules of the diffusing liquid is small compared to the characteristic geometric dimensions of the biotissue structural elements, so that according to the Stokes-Einstein theory [27] the immersion agent diffusion coefficient is determined only by the molecular sizes and the viscosity coefficient of the medium. Hence, the geometric factors of the biotissue strongly affect only the optical parameters of the sample (determining its light attenuation coefficient) and, at the same time, these parameters weakly affect directly the kinetics of the immersion fluid diffusion. Thus, the dependence of the biotissue immersion clearing kinetics on the geometric parameters of the biotissue structure is determined only the dependence of the light attenuation coefficient of the sample upon these factors. 


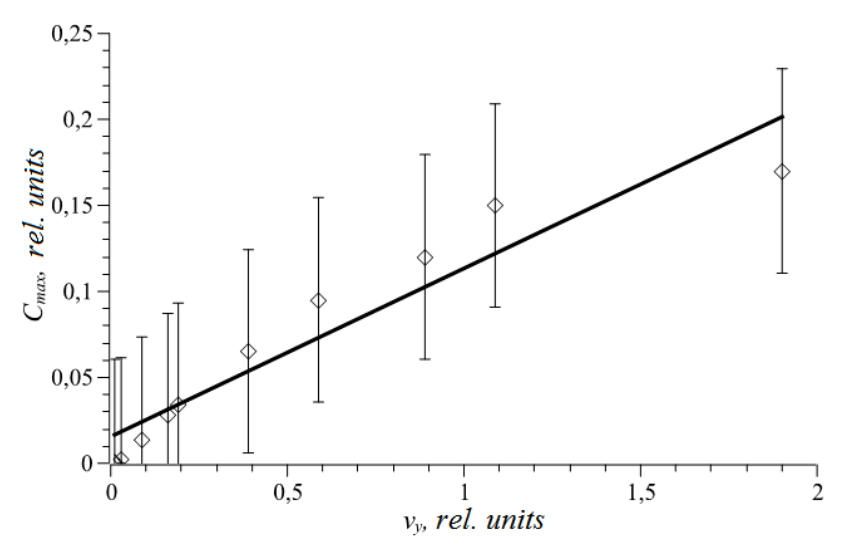

a

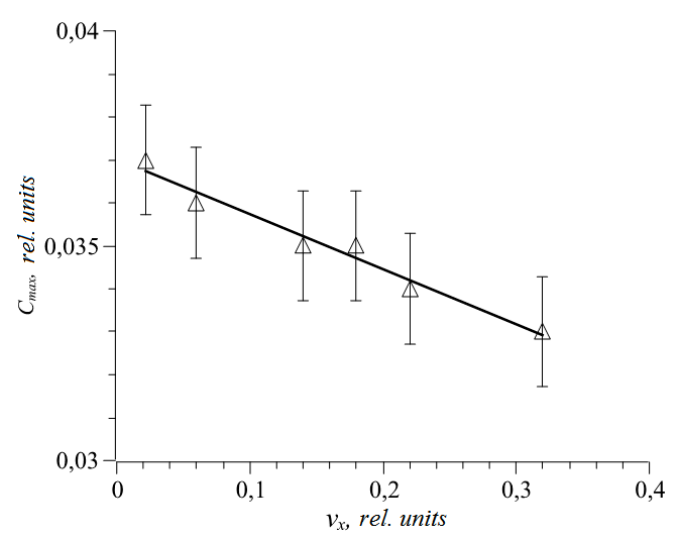

b

Fig. 2 The concentration of the fluid diffusing into the biotissue versus the diffusion rate (a - in the transverse direction, $C_{\max }=b+a v_{y} ; b=0.01 \pm 0.008 ; a=0.1 \pm 0.01 ; \mathrm{b}-$ in the longitudinal direction, $C_{\max }=b+a v_{x}$; $b=0.037 \pm 0.0002 ; a=-0.0128 \pm 0.0011)$.

\section{Conclusion}

We presented the experimental and theoretical results of the study of optical and diffusion properties of the cartilage tissue after the immersion clearing procedure and without it. These results can be summarised as follows.

1) The immersion impact on the cartilage tissue leads to its structural changes, increasing the size of the scattering elements, which agrees with the results of Ref. [25].

2) The immersion impact on the cartilage tissue leads to the change of its transmission spectrum in the infrared range. In the visible range, we observed no changes except the increased transmission in the samples subjected to immersion.

3) The immersion impact on the cartilage tissue does not change its refractive index anisotropy. The obtained estimate of the cartilage tissue refractive index anisotropy corresponds to the data of other papers $[19$, 24].

4) The kinetics of the immersion fluid diffusion in the biotissue strongly depends on the anisotropy of the diffusion rate along the collagen fibres and in the perpendicular direction. The increase of the mean diffusion rate of the immersion fluid in the biotissue under the local application can lead to the fall of light transmission rather than its expected growth.

\section{Disclosures}

All authors declare that there is no conflict of interests in this paper.

\section{Acknowledgments}

The author is grateful to E.A. Genina, (N.G. Chernyshevky National Research Saratov State University) for valuable recommendations on the use of Omnipaque in optical studies. The author also thanks M.I. Shishkina (N.G. Chernyshevky National Research Saratov State University) for providing the possibility of using the spectral system based on the MDR-41 monochromator.

The work was carried out under the financial support from the Russian Foundation for Basic Research, grant No. 15-29-04810 ofi_m. 